(6) OPEN ACCESS

\title{
Qualitative analysis of the experiences of gay, bisexual and other men who have sex with men who use GetCheckedOnline.com: a comprehensive internet-based diagnostic service for HIV and other STIs
}

\author{
Rod Eric Knight, ${ }^{1,2}$ Cathy Chabot, ${ }^{3}$ Anna Carson, ${ }^{1,2}$ Kimberly Thomson, ${ }^{3,4,5}$ \\ Devon Haag, ${ }^{4}$ Mark Gilbert, ${ }^{3,4}$ Jean Shoveller ${ }^{3}$
}

${ }^{1}$ British Columbia Centre on Substance Use, Vancouver, Canada

${ }^{2}$ Department of Medicine, University of British Columbia, Vancouver, Canada ${ }^{3}$ School of Population and Public Health, University of British Columbia, Vancouver, Canada ${ }^{4}$ British Columbia Centre for Disease Control, Vancouver, Canada

${ }^{5}$ Department of Psychology, Faculty of Health, Deakin University, Melbourne, Australia

\section{Correspondence to}

Dr Rod Eric Knight, British Columbia Centre on Substance Use, Vancouver V6Z 2A9, Canada; bccsu-rk@bccsu.ubc.ca

Received 9 April 2018 Revised 7 November 2018 Accepted 25 November 2018 Published Online First 13 January 2019

\section{Linked}

- http://dx.doi.org/10.1136/ sextrans-2017-053325

- http://dx.doi.org/10.1136/ sextrans-2018-053652

\section{Check for updates}

(C) Author(s) (or their employer(s)) 2019. Re-use permitted under CC BY-NC. No commercial re-use. See rights and permissions. Published by BMJ.

To cite: Knight RE, Chabot C, Carson A, et al. Sex Transm Infect 2019:95:145-150.

\section{ABSTRACT}

Objectives To describe the factors that influence gay, bisexual and other men who have sex with men's (gbMSM) experiences with GetCheckedOnline.com (GCO) in British Columbia (BC), Canada. GCO clients complete an internet-based risk assessment and print a laboratory test requisition form for HIV and other STIs to take to a private laboratory for diagnostic services.

Methods Drawing on a purposive stratified sampling framework, we conducted 37 in-depth semistructured interviews with gbMSM who had used GCO at least once between 2015 and 2017.

Results Participants expressed a preference for GCO (instead of clinic-based testing) because of convenience, privacy and control over specimen collection (specifically with doing one's own throat or anal swab). Participants preferred receiving their results online via GCO compared with phone or email follow-up by clinic staff. GCO was viewed positively because it offers gbMSM living outside of urban city centres easy access to diagnostic services, including access to pooled nucleic acid amplification testing. Many participants also continued to positively view the clinic-based services available for gbMSM in their community. These services were frequently described as highly competent, tailored and comprehensive in responding to more complex needs. For example, attending a clinic was viewed as preferential to GCO in instances where there was a desire to access services addressing co-occurring health issues (eg, mental health; substance use disorders). Almost all of the participants anticipated using both GCO and clinic-based services in the future.

Conclusions gbMSM report positive experiences and perceptions of GCO; however, they do not view GCO as a panacea. The results of this study point to the need to ensure that a wide range of integrated service options (eg, online; clinic-based) are available to address the range of sexual health needs of gbMSM living in BC's diverse settings.

\section{INTRODUCTION}

Gay, bisexual and other men who have sex with men (gbMSM) are a priority population for intervention due to disproportionately high rates of HIV and other STIs. Unfortunately, gbMSM also face many social and structural barriers to clinic-based STI diagnostic services. ${ }^{1-6}$ For example, previous research has identified how access to non-judgemental healthcare providers, ${ }^{5}$ convenient clinic hours and confidential or anonymous services ${ }^{5} 6$ represent key factors that influence gbMSM's decisions to get tested. Internet-based sexual health interventions can help address barriers experienced by gbMSM to clinic-based testing ${ }^{1-11}$ and have recently been implemented in select international settings, including several jurisdictions in British Columbia (BC), Canada.

After intensive consultations and research with key stakeholders (eg, healthcare professionals, community members) in $\mathrm{BC},,^{3} 4^{12}$ the $\mathrm{BC}$ Centre for Disease Control (BCCDC) launched GetCheckedOnline.com (GCO)-a comprehensive internet-based STI diagnostic service-in September 2014 in Vancouver, BC. See Gilbert et $a l^{11}$ for more information and figure 1 for further details about the process. (Full details on the service are published elsewhere). ${ }^{3}$

While early stakeholder consultations and evaluations have been very helpful for GCO's design and early implementation phases, ${ }^{3-5} 12$ there is limited research examining the experiences of users of internet-based STI diagnostic services. For example, previous research involving internet-based Chlamydia testing services has focused on receiving results online ${ }^{14}$ and on pathways to treatment post diagnosis after using online services. ${ }^{15}$ The objective of the current study is therefore to describe the factors that influence gbMSM's experiences with and preferences for both GCO and clinic-based testing.

\section{METHODS}

\section{Study setting}

We conducted in-depth semistructured interviews with gbMSM who had used GCO at least once. (For more information about study setting, please see Gilbert et $a l^{11}$ ). 


\section{How does T GETCHECKED work?}

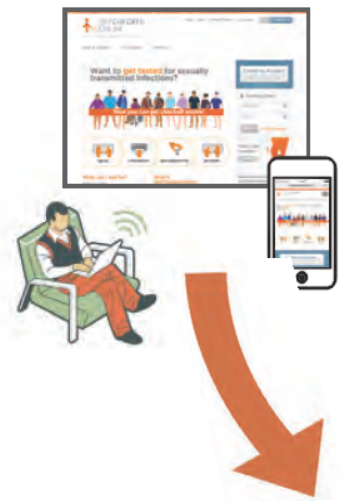

\section{Create an account on getcheckedonline.com}

A person enters an access code to create an account, which requires providing a name, gender and sex, and date of birth. Other information is optional.

Questions

are asked to make sure the right tests are offered and the right health information is given. Then the user prints a lab form or saves it to their smartphone.

People who have symptoms, or have a partner with an STI, are advised to get tested in a clinic.

\section{Tesł again!}

The next time users want to get tested, they can log back in and start the cycle all over again.
Get Checked Online is a service of the $B C$ Centre for Disease Control, in partnership with the BC Public Health Laboratory and Regional Health Authorities.

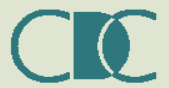

If you have any questions or comments, please email us at getcheckedonline@bccdc.ca

\section{Give samples}

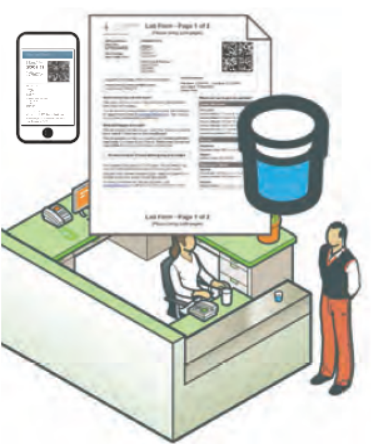

At the lab, users give their lab form or show their phone for scanning.

Users don't need to show ID.

\section{BC Centre for Disease Control}

\section{Get your results}
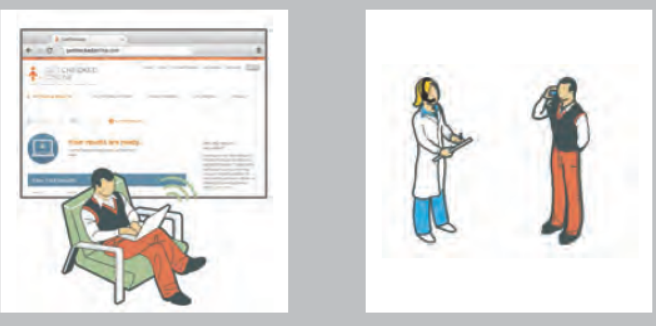

Users get an email when your results are ready. If all tesis are negative, users can see them online. Otherwise, users are asked to call the STI clinic to speak with a nurse about your fest results.

Figure 1 How does GetCheckedOnline work? 
In $\mathrm{BC}$, clinic-based testing is offered through publicly funded sexual health clinics, non-governmental organisations (including gbMSM-specific clinics in Vancouver), primary care programmes and public health clinics, although the availability of clinic-based testing varies in each community. In Vancouver and Victoria, for example, some testing services are available on evenings and weekends but wait times can be lengthy. Face-to-face testing in Kamloops is more limited, with testing only available during weekday business hours through family physicians, walk-in clinics and the university's student health centre. There is virtually no STI testing available through Kamloops' public health clinics unless individuals are street-involved.

\section{Data collection and analysis}

From October 2015 to May 2017, study participants were recruited through emails sent by the BCCDC after they had registered for a GCO account and consented to participate in GCO research. Drawing on a purposive stratified sampling framework, ${ }^{16}$ participants of varying social positions and lived experiences were recruited, including men from a range of socioeconomic statuses and sexual identities. To be eligible, participants had to identify as a cisgender or transgender man; report being sexually active with another man or men; be $\geq 15$ years of age; be English-speaking and have completed at least part of the GCO testing process (eg, some individuals received a testing requisition but did not go for specimen collection). The BCCDC's recruitment email asked prospective participants to contact the study's coordinator to confirm their eligibility and schedule an interview. Participants did not know the interviewers prior to recruitment and were informed that they were paid research staff.

Interviews were conducted by one of five experienced research staff (interviewers included co-authors CC (MA), KT $(\mathrm{MSc})$ and $\mathrm{AC}(\mathrm{MSc})$ and all were research coordinators at the time of data collection) in private offices or by phone. Participants had the choice of being interviewed by a woman or man. Participants were informed that the study's aim was to understand how clinic-based and internet-based testing services could better meet their needs. Participants completed an informed consent form and short sociodemographic questionnaire prior to the interview. Interviews ranged from $35 \mathrm{~min}$ to 2 hours; participants received an honorarium of $\$ 25-30$ (the amount was increased in 2016). Interviews were audio-recorded and transcribed verbatim (participants had the option of memberchecking their own transcripts) and transcripts were uploaded to NVivo V.11. ${ }^{17}$

The research team employed thematic analysis ${ }^{18}$ to develop an initial set of codes, which were developed through a combination of deductive and inductive approaches. ${ }^{19}$ For example, during the initial stage of analysis, raw data was coded lineby-line. We then moved into a broader coding scheme and codes focused on participants' experiences with sexual healthcare-seeking. Data collection and analysis were conducted iteratively, with later interviews helping identify gaps in our sample and inform subsequent data collection, as well as informing our assessments of data saturation. In later stages of analysis, interview transcripts and notes were re-read to examine how various social positions (eg, age, sexual identity) affected gbMSM's preferences for online or clinic-based testing. Two themes emerged around preference for testing mode: 'Convenience, Privacy and Control' (GCO) and 'Competent Service Provision, Tailored Care and Comprehensive Care' (clinic-based), presented below.

\begin{tabular}{|c|c|}
\hline Participants & $\mathrm{n}=37$ \\
\hline \multicolumn{2}{|l|}{ Age } \\
\hline (average, range) & $37.9(23-71)$ years \\
\hline \multicolumn{2}{|l|}{ Ethnicity* } \\
\hline White & $28(75.7 \%)$ \\
\hline Chinese & $2(5.4 \%)$ \\
\hline White+Filipino & $1(2.7 \%)$ \\
\hline Chinese +South East Asian & $1(2.7 \%)$ \\
\hline Arab/West Asian & $1(2.7 \%)$ \\
\hline Filipino & $1(2.7 \%)$ \\
\hline South Asian & $1(2.7 \%)$ \\
\hline Latin American & $1(2.7 \%)$ \\
\hline Indigenous & $0(0 \%)$ \\
\hline Black & $0(0 \%)$ \\
\hline \multicolumn{2}{|l|}{ Sexual identity } \\
\hline Gay & $27(72.9 \%)$ \\
\hline Bisexual & $7(18.9 \%)$ \\
\hline Straight & $1(2.7 \%)$ \\
\hline 'Hetero-flexible' & $1(2.7 \%)$ \\
\hline Queer & $1(2.7 \%)$ \\
\hline \multicolumn{2}{|l|}{ Born in Canada } \\
\hline Yes & $22(59.5 \%)$ \\
\hline No & $15(40.5 \%)$ \\
\hline \multicolumn{2}{|l|}{ Education } \\
\hline High school or less & $5(13.5 \%)$ \\
\hline College/undergraduate & $19(51.4 \%)$ \\
\hline Graduate or more & $7(18.9 \%)$ \\
\hline Missing & $6(16.2 \%)$ \\
\hline \multicolumn{2}{|l|}{ Testing frequency } \\
\hline Approximately every 3 months & $15(40.5 \%)$ \\
\hline Approximately every 6 months & $11(29.7 \%)$ \\
\hline Approximately every year & $2(5.4 \%)$ \\
\hline Other* & $5(13.5 \%)$ \\
\hline No data & $4(10.8 \%)$ \\
\hline \multicolumn{2}{|l|}{ Most recent testing experience } \\
\hline GCO & $28(76 \%)$ \\
\hline Clinic-based & $9(24 \%)$ \\
\hline \multicolumn{2}{|l|}{ Most recent testing experience with GCO } \\
\hline Complete testing & $31(84 \%)$ \\
\hline Created testing requisition but did not complete testing & $6(16 \%)$ \\
\hline
\end{tabular}

*'Other' includes answers which are not temporal (eg, 'after a "risky" encounter'; 'every 6-7 partners').

GCO, GetCheckedOnline.com.

\section{RESULTS}

We interviewed 37 participants, 33 of whom reported previously using clinic-based testing before having used GCO (250 invitation emails were sent out, four participants did not provide this information, no participants dropped out). Sample demographics are presented in table 1 . Below, we present our findings in two thematic sections and use quotes to illustrate key aspects of our analysis.

Preferences for online testing: convenience, privacy, control Many participants emphasised that they preferred using GCO to clinic-based testing because it was more convenient, expedient and timely. For example, based on their previous sexual healthcare experiences, many said they were aware of the sexual risks they engaged in and therefore viewed the counselling component 
of clinic-based testing as unnecessary. As such, these participants seemed to favour the immediacy and control that they associated with using GCO over the use of clinic-based testing. For example, one 29-year-old gay Vancouver man described:

I can [test with GCO] and get it done and get it booked basically as I think about it. I don't have to try to wait to do it later that day and then forget about it or that kind of thing. Yeah, it's convenient and it's more or less immediate. (GCO-GBMSM-037)

Several participants described how GCO provided an enhanced sense of privacy and offered a way to test without having to disclose one's sexual identity or behaviour to a healthcare provider. For example, a 71-year-old gay man from a Vancouver suburb described:

\begin{abstract}
'Cause you're there [clinic] to get help, you're there to get some assistance, not to be criticised for what you do. [...] Some people might [...] want to just stay as anonymous as possible. If I have a way of going online and finding out for myself where I stand with this, that's better for me. (GCO-GBMSM-012)
\end{abstract}

Several described how GCO's specimen collection protocol provided them with more autonomy and control than they experienced with clinic-based testing. For instance, several participants described how administering their own anal and/or throat swabs allowed them to avoid having potentially 'invasive' procedures performed within clinic-based settings. One 30-year-old gay Victoria man said:

I love that they have the swab ability in the lab because that was always something ... Getting blood drawn-it's not too bad. It's not really an invasion of privacy. But when you're being swabbed in private [body] areas, that's something that you'd probably rather do yourself if you can. (GCO-GBMSM-023)

Participants from areas outside of the urban city centres (eg, Vancouver suburbs) described how GCO provided them with a highly specialised sexual healthcare service that would not otherwise be available to them. Several participants described how they had previously relied on their family doctors to provide HIV testing and those experiences rarely were gbMSM-centred. For example, one 32-year-old gay man from a Vancouver suburb described how he frequently sought testing shortly after having sex that he felt was 'risky', so he would request the pooled Nucleic Acid Amplification Test (NAAT), which has the capacity to detect HIV 10-12 days after infection. While his family doctors tended not to be aware of the technical specificities of the NAAT, GCO offered him the ability to test using the pooled NAAT 'on demand'. As such, this participant described why he preferred GCO:

[GCO] do the pooled NAAT, the best test. So that's why [prefers GCO], because every time before GetCheckedOnline I had to explain to all my family doctors, 'I need to get the pooled NAAT because it picks up the bug, like, very early. It's the best test.' And they don't know what that is. So the family doctors are not educated. Now I don't need to do anything. I just go online and that's it. (GCO-GBMSM-020)

Many participants described how GCO changed their testing frequency. For example, participants described how GCO's regular testing reminder emails impacted both their testing frequency and understandings around corresponding risk. For instance, a 50-year-old gay Vancouver man described:

[GCO] reminds me for next time I have to be tested. And it did open my mind that, you know, hey, maybe you should care more about STDs happening and everything like that. So it kind of enlightened me or woke me up [laughs]. Yeah, 'Don't be so stupid, don't be so naïve, don't ...' And that was a good thing. (GCO-GBMSM-011)

Many participants also preferred how GCO provided them with more control around how they receive their results, particularly negative results. For example, several participants said their previous clinic-based testing experiences tended to leave them wondering if either the tests had not yet been processed or if the absence of a follow-up call from the clinic meant their results were negative. With GCO, participants described how having access to their results online allowed them to view a confirmed negative result, thereby alleviating any uncertainty and/or anxieties about a lack of follow-up. As one 27-year-old gay man from Vancouver described:

I got the results within a normal time and the fact that I was able to just look it up myself and know without having to wait for a 'noncall', it was excellent. It was all-round just game-changing. I just love it. (GCO-GBMSM-027)

Participants also described GCO as a complement to the clinic-based care they receive, not a replacement. For example, several said that they would no longer require regular testing from their family doctor. Nevertheless, these participants tended to view the care they receive via GCO as being integrated with the clinic-based services they receive; as one 62-year-old gay Kamloops man described:

I intend to use [GCO] all the time and I've already told my doctor that's what I'm going to be doing. So he said, 'Fine. That's great. That's really good.' So I'll go to him for my other blood work, for cholesterol and all that stuff, my overall physical. And, obviously if I did test positive for anything then I would go and see my doctor. (GCO-GBMSM-014)

\section{Preferences for clinic-based testing: competent, tailored, comprehensive}

A subset of participants said they would prefer clinic-based testing, including because some services are not available through GCO (eg, HIV rapid testing). Unlike those who preferred doing their own swabs, several indicated they would prefer that a clinician perform the procedure. For example, a 28-year-old gay Vancouver man who is a nurse described how he felt that self-collecting a throat swab could be too complicated if it is not clearly explained to those who are unfamiliar with self-collection procedures:

The throat swab that they end up doing for STIs can actually be a bit complicated. Pretty complicated instructions for-what you're essentially doing is just a really quick swab around the back of the uvula, snapping it, and throwing it back in the bag. But they [the laboratory staff] do kind of just hand it to you and say, you know, 'Here's your form, the instructions are there and go nuts, we'll see you in 5 min.' (GCO-GBMSM-029)

Others stated that the need to test regularly with their family doctor made using GCO redundant. For example, one 36-year-old gay Vancouver man described how he needed to see his doctor for blood work regularly to have his Pre-Exposure Prophylaxis (PrEP) prescription renewed:

One of the peculiar benefits of being on PrEP is it's a very scheduled kind of thing because your prescription lasts for a certain time and to get the prescription renewed you have to go and do your blood work ahead of time. You know, and so that makes me less likely to use GetCheckedOnline. Because I am sort of wired into this other 'system'. (GCO-GBMSM-001) 
Several participants described how clinic-based services within Vancouver were the gold standard of care for gbMSM and they would therefore not choose to use GCO. For example, a 29-year-old gay Vancouver man said he prefers accessing gbMSM-centred sexual health services because they make him feel more comfortable than using GCO and going to a private lab for specimen collection:

The nurses that they select for [gbMSM-centred health clinics] are also selected for their ability to be compassionate and to try to put people at ease because they're used to having the specific discussions around HIV. [...] [LGBTQ clinics are] just a little bit more comfortable because ... well, I guess part of it is just everything is gay there too. Whereas it's much more clinical at the [lab]. (GCO-GBMSM-037)

Several participants described how GCO could result in 'missed opportunities' for those experiencing co-occurring psychiatric conditions (eg, depressive disorders, addiction) that can be interrelated to sexual health issues; as one 28-year-old bisexual participant from a Vancouver suburb explained:

[GCO is] an online form that has no interaction, right? And so even though it may suit your needs, you might have some other experiences or situations that could benefit from seeing somebody who's trained in the field, right? Like, this service doesn't identify if I have HIV anxiety. [...] This service doesn't identify if I'm-for lack of a better word-a sex maniac and should be tested frequently. $[\ldots]$ There are other, we'll say mental health issues [...] that can be further diagnosed from a person than an Internet form that cannot diagnose you. (GCO-GBMSM-032)

\section{DISCUSSION}

Our study-among the first to report on the actual experiences of internet-based STI testing users (eg, rather than focusing on prospective users) ${ }^{5} 101315$ 20-22 — describes factors influencing experiences and preferences among gbMSM regarding their decisions to use internet-based and clinic-based STI testing. As suggested by our team's recently published analysis of survey data with GCO users demonstrating greater barriers in accessing clinic-based services and related to encounters with healthcare providers, ${ }^{11}$ participants expressed a preference for GCO because of convenience and privacy, and they typically described regular testing behaviour (eg, every few months). Participants also described preferring to have control of specimen collection (swabs). Participants preferred receiving results online via GCO compared with clinic-based testing (eg, 'non-calls' for negative results). GCO also provided enhanced access to testing technologies, including pooled NAAT tests and automated testing reminders. Many participants continued to positively view the clinic-based services available for gbMSM in their community, and clinic-based services were frequently described as competent, tailored and comprehensive in responding to more complex needs or co-occurring health issues (eg, mental health disorders). Participants anticipated using a combination of GCO and clinic-based services in the future (eg, for both sexual and other health-related needs), thereby supporting previous claims that internet-based STI testing services can contribute to the spectrum of services that are needed to reduce barriers to care among diverse client groups. ${ }^{22}$

While gbMSM view GCO positively, these findings indicate that GCO is not regarded as a panacea, highlighting the importance of keeping GCO integrated and up to date with other facets of the evolving intervention 'landscape'. For example, the introduction of publicly funded PrEP since January 2018 for gbMSM at risk of HIV acquisition in $\mathrm{BC}^{23}$ may increasingly make GCO somewhat 'redundant' for those engaging with BC's evolving PrEP 'systems', as receiving ongoing access to PrEP requires testing through a physician every three months. Future research will be helpful in identifying how GCO and other areas of the sexual healthcare system can best be adapted for scale-up in mutually symbiotic ways (eg, integrated scale-up of GCO and the PrEP 'system'). For example, our findings provide support for the need to continue the ongoing implementation, adaptation and evaluation of GCO to better understand how internet-based services can begin to employ a 'syndemics' perspective ${ }^{24}$ (eg, the integration of mental health, harm reduction and substance use services) to respond to the needs of gbMSM experiencing co-occurring health issues (eg, online referral pathways). Finally, these findings demonstrate the importance of ongoing efforts to ensure general healthcare practitioners are aware of the different testing options in BC, including understandings of how GCO fits within their clinical practice and the testing technologies available in $\mathrm{BC}$ (eg, pooled NAAT).

These study findings also highlight how GCO may enhance gbMSM's testing behaviour (eg, frequency and timing), suggesting that continued efforts to scale-up GCO may help reduce sexual health inequities experienced by gbMSM in BC. However, these findings need to be interpreted with caution. For example, there are several limitations to this study design, including sampling, participation and social desirability biases. The study sample reflects a group of gbMSM who are generally knowledgeable about testing and/or 'frequent' testers. Future research should examine how GCO is perceived or 'taken up' by gbMSM with less testing experience and/or knowledge about STIs. Despite these limitations, our study provides important insights into identifying factors that influence gbMSM's experiences with and preferences for GCO and clinic-based testing.

\section{CONCLUSION}

These findings indicate that gbMSM report positive experiences and perceptions of GCO; however, gbMSM do not view GCO as a panacea and they tended to view it as being integrated with the clinic-based services they receive. The results of this study indicate the need to ensure that a wide range of integrated service options (eg, online; clinic-based; PrEP 'systems') are available to address the range of sexual health needs of gbMSM living in BC's diverse settings.

\section{Key messages}

- This is the first study to report results on the actual experiences of users of a comprehensive internet-based STI testing service.

- Gay, bisexual and other men who have sex with men (gbMSM) report positive experiences with GetCheckedOnline. com.

- gbMSM expressed a preference for GetCheckedOnline.com over clinic-based testing because of convenience, privacy and control.

- Internet-based testing services can contribute to the spectrum of services that are needed to reduce barriers to care among gbMSM.

\section{Handling editor Jackie A Cassell}

Acknowledgements The authors thank the study participants for sharing their experiences with them, and Kyle Sutherland and Caroline Mniszak for assisting with data collection. They also thank Natasha Parent and Henry Wu for assisting with coding of the data; and Caroline Mniszak for preparing table 1 for this manuscript. 
Contributors Conception and design of the study: JS, MG, RK and DH. Data collection: CC, AC, KT and RK. Data analysis and interpretation: RK, CC, AC, KT, DH, $M G$ and JS. Drafting manuscript: RK, CC, AC, KT, DH, MG and JS.

Funding This study was funded by the Canadian Institutes of Health Research (POH-106440, HIM-145-373, PHE-114129; PHE-318068; CTW-155550). Knight is supported by a Scholar Award from the Michael Smith Foundation for Health Research.

Competing interests None declared.

Patient consent for publication Not required.

Ethics approval This study received institutional ethics approval from the University of British Columbia's Behavioural Research Ethics Board (\# H12-02408 and H16-00846) and UBC's Clinical Research Ethics Board (\# H15-00510).

Provenance and peer review Not commissioned; externally peer reviewed.

Data sharing statement All relevant data are presented within the paper and are fully sufficient to replicate the study findings.

Open access This is an open access article distributed in accordance with the Creative Commons Attribution Non Commercial (CC BY-NC 4.0) license, which permits others to distribute, remix, adapt, build upon this work non-commercially, and license their derivative works on different terms, provided the original work is properly cited, appropriate credit is given, any changes made indicated, and the use is non-commercial. See: http://creativecommons.org/licenses/by-nc/4.0/.

\section{REFERENCES}

1 Sandstra IL, Gold F, Jones E, et al. Cyber outreach: ST/HIV education online. Can Nurse 2008;104:24.

2 Bauermeister JA, Pingel ES, Jadwin-Cakmak L, et al. Acceptability and preliminary efficacy of a tailored online HIV/STI testing intervention for young men who have sex with men: the Get Connected! program. AIDS Behav 2015;19:1860-74.

3 Gilbert M, Haag D, Hottes TS, et al. Get Checked... Where? The development of a comprehensive, integrated internet-based testing program for sexually transmitted and blood-borne infections in British Columbia, Canada. JMIR Res Protoc 2016:5:e186.

4 Gilbert M, Salway T, Haag D, et al. Use of GetCheckedOnline, a comprehensive web-based testing service for sexually transmitted and blood-borne infections. J Med Internet Res 2017;19:e81.

5 Hottes TS, Farrell J, Bondyra M, et al. Internet-based HIV and sexually transmitted infection testing in British Columbia, Canada: opinions and expectations of prospective clients. J Med Internet Res 2012;14:e41.

6 Wray T, Chan PA, Simpanen E. eTEST: developing a smart home hiv testing kit that enables active, real-time follow-up and referral after Testing. JMIR Mhealth Uhealth 2017;5:e62.

7 Mitchell JW, Torres MB, Joe J, et al. Formative work to develop a tailored hiv testing smartphone app for diverse, at-risk, hiv-negative men who have sex with men: a focus group study. JMIR Mhealth Uhealth 2016;4:e128-e28.
8 Horvath KJ, Bauermeister JA. Ehealth literacy and intervention tailoring impacts the acceptability of a HIV/STI testing intervention and sexual decision making among young gay and bisexual men. AIDS Educ Prev 2017;29:14-23.

9 Lampkin D, Crawley A, Lopez TP, et al. Reaching suburban men who have sex with men for std and hiv services through online social networking outreach: a public health approach. J Acquir Immune Defic Syndr 2016;72:73-8.

10 Knight R, Karamouzian M, Salway T, et al. Online interventions to address HIV and other sexually transmitted and blood-borne infections among young gay, bisexual and other men who have sex with men: a systematic review. J Int AIDS Soc 2017;20:e25017.

11 Gilbert M, Thomson K, Salway T, et al. Differences in experiences of barriers to STI testing between clients of the internet-based diagnostic testing service GetCheckedOnline.com and an STI clinic in Vancouver, Canada. Sex Transm Infect 2018:sextrans-2017-053325.

12 Gilbert M, Bonnell A, Farrell J, et al. Click yes to consent: Acceptability of incorporating informed consent into an internet-based testing program for sexually transmitted and blood-borne infections. Int J Med Inform 2017;105:38-48.

13 Shoveller J, Knight R, Davis W, et al. Online sexual health services: examining youth's perspectives. Can J Public Health 2012:103:14-18.

14 Gibbs J, Aicken CRH, Sutcliffe LJ, et al. Mixed-methods evaluation of a novel online ST results service. Sex Transm Infect 2018;94:622-4.

15 Aicken CRH, Sutcliffe LJ, Gibbs J, et al. Using the eSexual Health Clinic to access chlamydia treatment and care via the internet: a qualitative interview study. Sex Transm Infect 2018;94:241-7.

16 Palinkas LA, Horwitz SM, Green CA, et al. Purposeful sampling for qualitative data collection and analysis in mixed method implementation research. Adm Policy Ment Health 2015:42:533-44.

17 QSR International. NVivo 11 for Windows, 2017.

18 Fereday J, Muir-Cochrane E. Demonstrating rigor using thematic analysis: a hybrid approach of inductive and deductive coding and theme development. Int I Qual Methods 2006:5:80-92.

19 Bernard H. Research methods in anthropology: qualitative and quantitative approaches. 4 edn. Lanham, MD: AltaMira, 2006.

20 Lorimer K, McDaid L. Young men's views toward the barriers and facilitators of Internet-based Chlamydia trachomatis screening: qualitative study. J Med Internet Res 2013:15:e265.

21 Tomnay JE, Bourke L, Fairley CK. Exploring the acceptability of online sexually transmissible infection testing for rural young people in Victoria. Aust J Rural Health 2014;22:40-4

22 Wellings K, Mehl GL, Free CJ. eSexual health interventions: promising, but more evidence needed. Lancet Public Health 2017:2:e162-e163.

23 British Columbia Centre for Excellence in HIV/AIDS. HIV Pre-Exposure Prophylaxis 2018. 2018. Available: http://cfenet.ubc.ca/hiv-pre-exposure-prophylaxis-prep [Accessed Mar 22 2018]

24 Singer M, Bulled N, Ostrach B, et al. Syndemics and the biosocial conception of health. The Lancet 2017;389:941-50. 